\title{
BMJ Open A randomised controlled trial of a consumer-focused e-health strategy for cardiovascular risk management in primary care: the Consumer Navigation of Electronic Cardiovascular Tools (CONNECT) study protocol
}

\author{
Julie Redfern, ${ }^{1}$ T Usherwood, ${ }^{2}$ M F Harris, ${ }^{3}$ A Rodgers, ${ }^{1} \mathrm{~N}$ Hayman, ${ }^{4} \mathrm{~K}$ Panaretto, ${ }^{5}$ \\ C Chow, ${ }^{1}$ A Y S Lau, ${ }^{6}$ L Neubeck, ${ }^{7}$ G Coorey, ${ }^{8}$ F Hersch, ${ }^{9}$ E Heeley, ${ }^{1}$ A Patel, ${ }^{1}$ \\ $\mathrm{S}$ Jan, ${ }^{1} \mathrm{~N}$ Zwar, ${ }^{10} \mathrm{D}$ Peiris ${ }^{1}$
}

To cite: Redfern J, Usherwood T, Harris MF, et al. A randomised controlled trial of a consumer-focused e-health strategy for cardiovascular risk management in primary care: the Consumer Navigation of Electronic Cardiovascular Tools (CONNECT) study protocol. BMJ Open 2014;4:e004523. doi:10.1136/bmjopen-2013004523

- Prepublication history for this paper is available online. To view these files please visit the journal online (http://dx.doi.org/10.1136/ bmjopen-2013-004523)

Received 21 November 2013 Accepted 13 December 2013

CrossMark

For numbered affiliations see end of article.

Correspondence to

Dr Julie Redfern; jredfern@georgeinstitute. org.au

\section{ABSTRACT}

Introduction: Fewer than half of all people at highest risk of a cardiovascular event are receiving and adhering to best practice recommendations to lower their risk. In this project, we examine the role of an e-health-assisted consumer-focused strategy as a means of overcoming these gaps between evidence and practice. Consumer Navigation of Electronic Cardiovascular Tools (CONNECT) aims to test whether a consumer-focused e-health strategy provided to Aboriginal and Torres Strait Islander and non-indigenous adults, recruited through primary care, at moderate-to-high risk of a cardiovascular disease event will improve risk factor control when compared with usual care.

Methods and analysis: Randomised controlled trial of 2000 participants with an average of 18 months of follow-up to evaluate the effectiveness of an integrated consumer-directed e-health portal on cardiovascular risk compared with usual care in patients with cardiovascular disease or who are at moderate-to-high cardiovascular disease risk. The trial will be augmented by formal economic and process evaluations to assess acceptability, equity and cost-effectiveness of the intervention. The intervention group will participate in a consumer-directed e-health strategy for cardiovascular risk management. The programme is electronically integrated with the primary care provider's software and will include interactive smart phone and Internet platforms. The primary outcome is a composite endpoint of the proportion of people meeting the Australian guideline-recommended blood pressure (BP) and cholesterol targets. Secondary outcomes include change in mean BP and fasting cholesterol levels, proportion meeting $\mathrm{BP}$ and cholesterol targets separately, self-efficacy, health literacy, self-reported point prevalence abstinence in smoking, body mass index and waist circumference, self-reported physical activity and self-reported medication adherence.

Ethics and dissemination: Primary ethics approval was received from the University of Sydney Human

\section{Strengths and limitations of this study}

In this project, we will examine the role of an integrated e-health consumer strategy as a means of overcoming such health system inefficiencies.

- The Consumer Navigation of Electronic Cardiovascular Tools (CONNECT) study will generate rigorously evaluated findings on an issue of national and international importance.

- The development of a content-rich, consumerfocused e-health intervention that is fully integrated with primary healthcare system will greatly inform the e-health agenda.

- If effective, the CONNECT e-health strategy could be upscaled and expanded to increase compliance with international e-health strategies. The CONNECT strategy may also have applicability as a standalone strategy where electronic integration with primary care is unavailable.

- Potential limitations are that this is an Australian study and relies on integration with the Australian personally controlled e-health record. In addition, the intervention is primarily consumer driven.

Research Ethics Committee and the Aboriginal Health and Medical Research Council. Results will be disseminated via the usual scientific forums including peer-reviewed publications and presentations at international conferences

Clinical Trials registration number: ACTRN12613000715774.

\section{INTRODUCTION}

Cardiovascular disease burden

Cardiovascular disease (CVD), including coronary heart disease and stroke, is the leading 
cause of death and disease burden globally. ${ }^{1}$ Importantly, in Australia, Aboriginal and Torres Strait Islander peoples experience approximately five times greater CVD burden than other Australians. ${ }^{2}$ Fewer than $50 \%$ of adults who attend Australian general practice or Aboriginal Community Controlled Health Services (ACCHSs) are adequately screened for vascular risk, and for those identified at high risk, only about $40 \%$ are receiving best practice care. ${ }^{3-5}$ Similar findings have been noted in other Australian studies. ${ }^{6-9}$ Adherence rates to lifestyle modification are around $30 \%^{10}$ and adherence to recommended medicines may be as low as $50 \%$ after 6 months of therapy. ${ }^{11}$ Overall, these studies have demonstrated failure to adequately implement effective interventions (that are also adhered to) to lower CVD risk for those who need it most.

\section{Consumer-focused e-health interventions}

During the past decade, there has been rapid development in consumer e-health. Several studies have shown the benefits of interactive Internet portals for managing chronic conditions (asthma, ${ }^{12-14}$ type 2 diabetes, ${ }^{15}$ arthritis, ${ }^{16}$ hypertension ${ }^{17}$ and mental health ${ }^{18}{ }^{19}$ ), for health behaviour change ${ }^{20} 21$ and lifestyle risk factors (physical activity, ${ }^{22}$ smoking cessation ${ }^{23}$ and weight loss). ${ }^{24}$ A Cochrane review of 124 studies concluded that computer-based 'Interactive Health Communication Applications' can also improve cognitive and social outcomes of patients with chronic conditions. ${ }^{25}$ The emerging evidence on text message interventions also appears promising. Five randomised controlled trials (RCTs) have demonstrated the effectiveness of mobile phone text messaging to promote smoking cessation, ${ }^{26} 27$ and a number of small RCTs have shown improved outcomes related to weight loss, ${ }^{28}$ physical activity, ${ }^{29}$ asthma medication adherence, ${ }^{30}$ glycaemic control in diabetes, ${ }^{31}$ blood pressure (BP) lowering, ${ }^{32}$ liver transplantation ${ }^{33}$ and HIV treatment. ${ }^{34}$ Other studies looking at text messaging systems to improve adherence to lifestyle and medication recommendations for people with established coronary artery disease are ongoing. ${ }^{35} 36$

Although the evidence for consumer-focused interventions is promising, there are few randomised evaluations of personally controlled e-health records (PCEHRs). ${ }^{37}$ The PCEHR is the Australian secure online summary of an individual's health information. This e-health record allows people, their doctors, hospitals and other healthcare providers to view and share health information and ideally improve care. Most studies investigating online personal health records have had small sample sizes and short follow-up periods ( $<12$ months). This raises concerns about their external validity and sustainability. One Australian system which features a personal health record, tools to assist with making decisions, organising tasks and a social networking feature has been shown in a community-based trial to promote improved uptake of influenza vaccination among university students. ${ }^{38}$ We are not aware of any RCTs that have incorporated multiple e-health components as part of a multifaceted, complex intervention for chronic disease management and prevention. Critically, there are no trials involving Aboriginal and Torres Strait Islander peoples, and attempts to integrate consumer strategies with Australian primary healthcare electronic health records are at a very early stage.

\section{Digital technologies and access}

Equitable digital access is critical to implementation of the national e-health strategy. Digital access is growing exponentially in Australia. In 2010-2011, 79\% of Australian households had access to the Internet in their home and $77 \%$ of these used the Internet daily. ${ }^{39}$ Among Aboriginal and Torres Strait Islander adults, in 2008, 59\% had Internet access (up from $41 \%$ in 2002) ${ }^{40}$ Mobile phone use has been dramatically rising and now outstrips computer access. In 2006-2007, 81\% of Australian consumers reported owning a mobile phone. Although growth was originally driven by the younger market, the largest annual increase in mobile phone ownership is among people aged $65+$ years. ${ }^{41}$ Data on mobile phone access for Aboriginal and Torres Strait Islander people are unclear; however, in 2008, $67 \%$ of non-remote and $61 \%$ of remote households had access to a prepaid mobile phone, and $41 \%$ and $19 \%$ of non-remote and remote households, respectively, had mobile access via a contract. ${ }^{41}$ 'Smart phones', characterised by multimedia and Internet connectivity, are the biggest growth area. In 2010-2011, around 25\% (3.9 million people) of Australians accessing the Internet did so via their phone (up $63 \%$ on the previous year). ${ }^{42}$

\section{HealthTracker e-health system}

An electronic patient care system called HealthTracker has been previously developed and tested by our research team. ${ }^{43}$ This e-health system is essentially a clinical decision support system for CVD risk management in primary care. HealthTracker is fully integrated with the primary healthcare electronic health record and provides: (1) point of care decision support related to CVD prevention and management; (2) a graphical patient counselling tool; (3) a computerised audit tool that provides rapid snapshots on health service performance combined with a recall and reminder system and (4) access to a quality improvement web-based portal where health services can view monthly peer-ranked performance and access tools and resources to support quality care. HealthTracker has undergone significant proof of concept testing and validation, ${ }^{43-45}$ and is currently being implemented in a large-scale cluster RCT (TORPEDO) in Australia. ${ }^{42}$ The trial is funded by the Australian National Health and Medical Research Council and involves 20 ACCHSs, 40 general practices and over 50000 patients (Grant ID \#1010547, ACTRN12611000478910). However, HealthTracker is a provider-directed strategy and the added value of a consumer or patient-focused interface remains unknown. 
Therefore, the Consumer Navigation of Electronic Cardiovascular Tools (CONNECT) study aims to enhance the existing HealthTracker system and utilise the growing e-health environment to scientifically test whether a consumer-focused e-health strategy provided to Aboriginal and Torres Strait Islander as well as nonindigenous people at moderate-to-high risk of a CVD event will improve risk factor control when compared with usual healthcare. The study also aims to determine the acceptability, equity and cost-effectiveness of such a strategy. We hypothesise that access to an integrated and patient-centred e-health strategy will improve risk factor control when compared with usual healthcare.

\section{Methods and analysis}

Study design

CONNECT is a single blind, RCT involving 2000 regular adult health service attendees at General Practices and ACCHSs with an average follow-up of 18 months (figure 1). Written and informed consent will be obtained from all participants.

The study will be conducted across approximately 65 Australian General Practices and ACCHSs. Participants with a diagnosis of CVD or who are at high risk of CVD will be randomly allocated to either the control or intervention group. The control group will continue to participate in usual healthcare, supported by HealthTracker; the intervention group will participate in the CONNECT programme which is a consumerdirected e-health strategy for cardiovascular risk management. The programme is electronically integrated with the primary care provider's software and will include access to interactive smart phone and Internet

\begin{tabular}{|c|c|}
\hline $\begin{array}{ll}\text { Pa } \\
\text { - } & \text { Moderate-high cardiovascula } \\
\text { - } & \text { Able to access the Internet at } \\
\text { Written, informed consent }\end{array}$ & $\begin{array}{l}\text { ility } \\
\text { nonthly }\end{array}$ \\
\hline $\begin{array}{l}\text { Rando } \\
\text { - } \quad \text { Baseline data collection (via } \\
\text { - } \quad \text { Straction from health record } \\
\text { - } \quad \text { Computer-generated random }\end{array}$ & $\begin{array}{l}=\mathbf{2 , 0 0 0}) \\
\text { visit and automated data } \\
\text { al status, cardiovascular risk level } \\
\text { llocation }\end{array}$ \\
\hline$\downarrow$ & 7 \\
\hline $\begin{array}{l}\text { CONNECT e-health strategy } \\
\quad \begin{array}{l}(\mathbf{n}=\mathbf{1 , 0 0 0}) \\
\text { - }\end{array} \\
\text { Access services in Figure } 2 \\
\text { Usual medical care for } \\
\text { medications and lifestyle }\end{array}$ & $\begin{array}{l}\text { Usual health-care } \\
(\mathbf{n = 1 , 0 0 0 )} \\
\text { - Usual medical care for } \\
\text { medications and lifestyle }\end{array}$ \\
\hline $\begin{array}{l}\text { Follow-up visits at } 12 \text { anc } \\
\text { - } \quad \text { Primary: Proportion of parti } \\
\text { pressure and lipid levels } \\
\text { - } \quad \text { Secondary: Health literacy, } \\
\text { activity, medication adherenc } \\
\text { - } \quad \text { Process and economic evalua }\end{array}$ & $\begin{array}{l}\text { dy (average of } 18 \text { months) } \\
\text { eting Australian guideline blood } \\
\text { sation, overweight, physical } \\
\text { scular and renal events. } \\
\text { of study }\end{array}$ \\
\hline
\end{tabular}

Figure 1 The Consumer Navigation of Electronic Cardiovascular Tools (CONNECT) study schema. platforms. Control arm participants will not have access to the portal. However, at the end of study, all participants (control and intervention) will be offered portal access for a maximum of 12 months. Participants will be assessed by personnel blinded to treatment allocation at face-to-face appointments at baseline and 12 months and at the end of study.

\section{Randomisation}

Eligible consenting participants will be randomly assigned to the e-health strategy or provision of usual care for an average of 18 months (minimum 12 months and maximum 24 months). In both groups, any advice and/or other interventions provided by the general practitioner (GP)/health service will continue at their health provider's discretion. Randomisation will be conducted independently using a central computer-based randomisation service with equal allocation to intervention versus control. A permuted block sequence will be used and will be stratified by level of CVD risk, study centre and Aboriginal and/or Torres Strait Islander status. Study personnel taking follow-up assessments will also be blinded to parallel group assignments.

\section{Participant eligibility}

Consenting adult patients ( $>18$ years) with access to the Internet at least once a month via mobile phone, tablet or computer who are at moderate-to-high risk of a CVD event will be included. Potential participants will be excluded if they have a severe intellectual disability or if they have insufficient English knowledge to provide written informed consent. Moderate-to-high CVD risk is defined as any of the following:

1. Five-year CVD risk $\geq 10 \%$ using the Framingham risk equation;

2. A clinically high risk condition (Aboriginal/Torres Strait Islander and age $>75$ years, diabetes and age $>60$ years, diabetes and albuminuria, epidermal growth factor receptor $<45 \mathrm{~mL} / \mathrm{min}$, systolic $\mathrm{BP}$ $\geq 180 \mathrm{~mm} \mathrm{Hg}$, diastolic $\mathrm{BP} \geq 110 \mathrm{~mm} \mathrm{Hg}$ and total cholesterol $>7.5 \mathrm{mmol})^{46}$;

3. An established CVD diagnosis (ischaemic heart disease, stroke/transient ischaemic attack and peripheral vascular disease).

\section{Recruitment}

We will aim to recruit approximately 30 participants from each of a total of 65 general practices and ACCHSs. Eligible patients will be identified by clinic staff using a customised electronic data extraction facility in the practice software system ${ }^{47}$ which was successfully used in the TORPEDO trial. ${ }^{43}$ Potential participants will receive an invitation letter from their GP. Eligibility will then be confirmed during a telephone call from a study research assistant. The interested individuals will be invited to a face-to-face registration visit at the practice from which they were identified. At the initial visit, written informed consent will be obtained and the 
baseline assessment will be completed by a CONNECT research assistant. Practices will be reimbursed a small fee (in line with a standard consultation fee) for the time required to assist with recruitment if the minimum of 25 patients per practice are recruited. All software license costs and technical support associated with the HealthTracker system will be provided free for the duration of the trial.

\section{Intervention}

The intervention group will participate in the CONNECT programme, which is a consumer-focused e-health strategy aimed at assisting with the management and prevention of CVD (figure 2). The programme components focus on cardiovascular risk assessment, medication adherence, lifestyle change and seamless patient-provider communication. CONNECT content has been informed by a detailed analysis of factors that drive uptake of Internet-based programmes for CVD secondary prevention. ${ }^{48}$ HealthTracker assessment data (eg, CVD risk factors and scores, medications and other treatment advice) are uploaded securely to a consumerfocused CVD-specific module built using a e-health record architecture. Patients will then be able to securely register for access to the integrated portal. Throughout the trial, a minimum of one upload from their software to the CONNECT portal will be performed by the GP or an authorised staff member for participants randomised to the intervention. Subsequent uploads to the CONNECT system will be at the discretion of the treating practitioner and will depend on visit frequency and availability of new data (based on changes in patient care and frequency of visits). An alert will be flagged in the application if a new upload has occurred. CONNECT was systematically developed through an iterative process and using user-centred design approach. ${ }^{49}$ The intervention development process involved collaborative design workshops (including journey mapping and persona building), ${ }^{49}$ sketching and iterative validation by consumers.

The portal will be accessible via the Internet and also via a downloadable application for use on a mobile device. As described in figure 2, participants in the intervention arm will be able to securely access the consumer portal via a secure login process. Patient data will be electronically uploaded from the clinic record to the portal. As part of the intervention, an implementation team of 'CONNECT coordinators' will provide face-to-face training to participants on how to use the various features in the portal. Key training features include: (1) viewing personal health record summary including information such as medicines, test results, BP and weight; (2) use of interactive tools and resources (eg, the HealthTracker risk calculator that visually plots CVD risk projections and allows people to perform 'what if scenarios' to explore the relative risk reductions (RRRs) from various CVD risk factors); (3) access to simple medication and healthy lifestyle reminders and motivational message prompts depending on their choice and health profile (eg, smokers wanting to quit

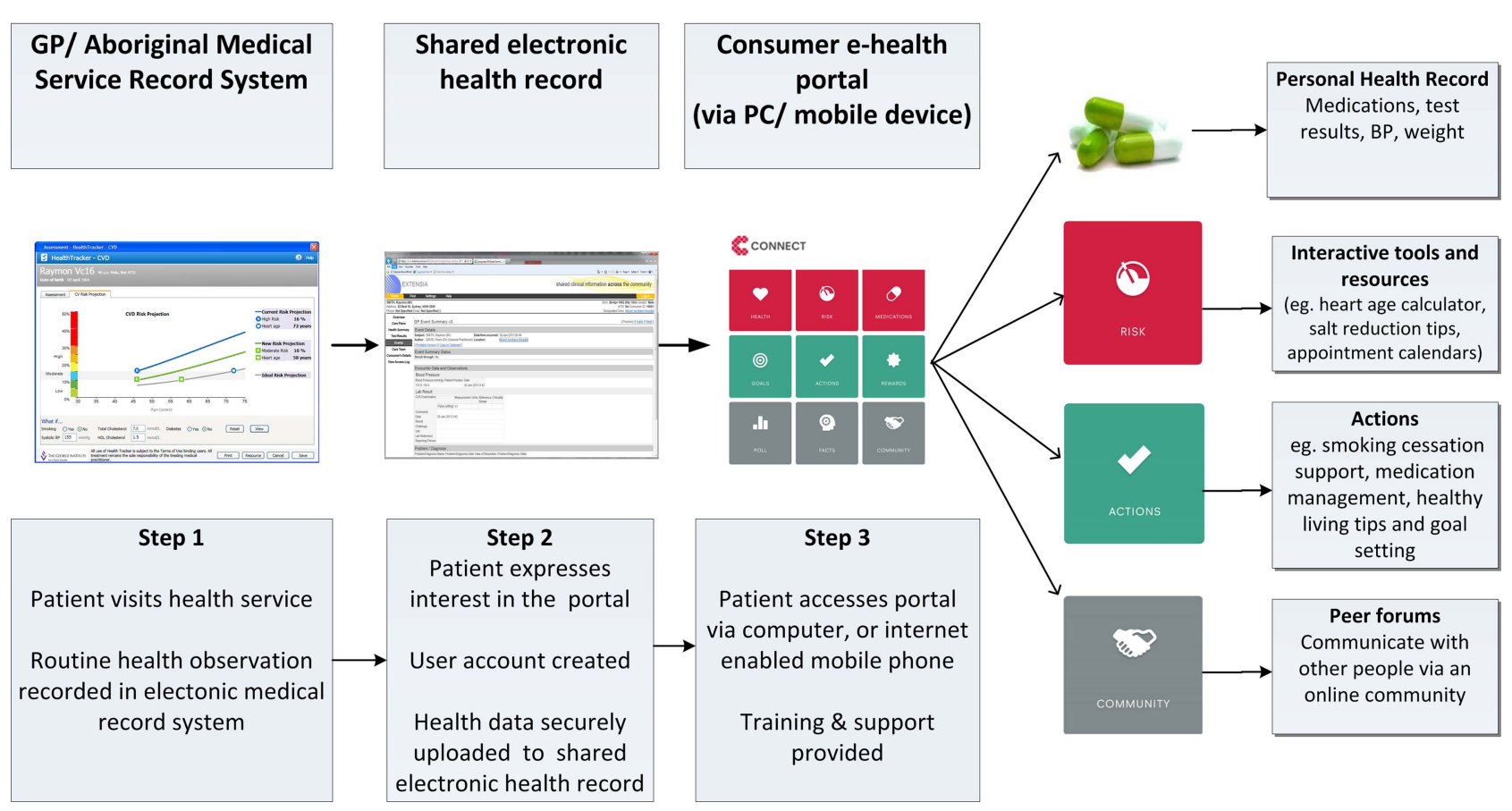

Figure 2 The consumer-focused e-health strategy linked to primary healthcare. 
will be able to receive a series of random messages to assist with cessation) and (4) an interactive goal setting and social media feature where people will be able to set their own goals and receive virtual rewards and also communicate with other users using CONNECT. As part of the intervention, participants will be contacted at months one and two by telephone and additional support will be provided as needed. At any time, assistance will also be available via a helpdesk number or via an online or mobile text help request. A CONNECT coordinator will receive these requests and provide appropriate medical support or arrange technical advice if needed. The CONNECT coordinators will be separate from the recruitment and assessment team so as to maintain blinding of outcome assessments.

\section{Control group}

Participants in the intervention and control groups will continue with usual healthcare. Control arm participants will not have access to the portal; however, at the end of study, all participants (control and intervention) will be offered portal access for a maximum of 12 months.

\section{Data collection and study outcomes}

Centrally employed CONNECT research assistants (blinded to group allocation) will conduct study visits at baseline, 12 and 24 months and ensure all clinical measures are entered into a purpose-built and secure online database. Clinical and survey data will be collected via face-to-face or phone interviews (if necessary) and entered into a secure central web-based database. During 12-month and 24-month assessments, we will assess the access to CVD management services for both groups such as the frequency of GP and specialist visits (self-report), access to allied health services (eg, dietitian and psychologists) and community groups or activities (eg, local walking group, online smoking cessation programme). In addition, analytic information from the CONNECT portal and smartphone application will be extracted on a monthly basis to determine usage patterns (website and smartphone/tablet application). This study will be monitored and managed centrally with periodic site monitoring visits.

The primary endpoint is the proportion of participants at the end of study whose BP and fasting lowdensity lipoprotein (LDL) cholesterol are meeting the Australian guideline targets (defined as: $\leq 130 / 80$ for participants with CVD, diabetes or albuminuria OR $\leq 140 / 90 \mathrm{~mm} \mathrm{Hg}$ for all others participants) and LDL-cholesterol $<2 \mathrm{mmol} / \mathrm{L}){ }^{50}$ Secondary outcomes include the mean difference from baseline in systolic and diastolic BP and fasting LDL cholesterol levels, proportion meeting BP and LDL targets separately, selfefficacy, health literacy, self-reported point prevalence abstinence in smoking, mean difference from baseline in body mass index and waist circumference, selfreported physical activity and self-reported medication adherence (box 1).
Box 1 Primary and secondary outcomes (measured at baseline, 12 months and end of study)

\section{Primary}

Proportion of participants meeting the Australian guideline BP and lipid targets ${ }^{50}$

- BP of $140 / 90 \mathrm{mmHg}$ for all except those with CVD, diabetes or albuminuria for whom the target BP is $130 / 80 \mathrm{mmHg}$. BP measurement is conducted via three resting and sitting digital recordings with the mean of the last two readings.

- $\mathrm{LDL}$ of $<2.0 \mathrm{mmol} / \mathrm{L}$. LDL measurement is via fasting blood sample.

BP is based, on an average, of three resting and sitting digital recordings with the mean of last two readings, and LDL-cholesterol is measured on a fasting blood sample.

\section{Secondary}

- Proportion meeting guideline-recommended BP and LDL-cholesterol targets separately

- Difference in mean systolic and diastolic BP at the end of study

- Difference in mean cholesterol levels at the end of study (TC LDL and HDL)

Difference in mean body mass index and waist circumference at the end of study

Difference in health literacy scores-HLQ ${ }^{51}$ and the eHEALS ${ }^{52}$ at the end of study

- Cardiovascular and renal events, new onset diabetes-self report and confirmed with medical records

- Physical activity-WHO Global Physical Activity Questionnaire ${ }^{53}$

- Point abstinence in smoking $(\leq 5$ cigarettes in the previous 7 days $^{54}$ or recent smoking according to assessment using carbon monoxide meter)

- Fruit and vegetable intake, fish, salt and saturated fat intakeself-report portions consumed in 7 days prior and compared with published guidelines recommendations

- Cardioprotective medication adherence-self-report and verified by medical record and pharmaceutical benefits scheme data

- All-cause mortality-medical record

- Hospital readmissions-self-report and verified by medical record

- Health-related quality of life-EQ5D (version 5L with Australian standardised weights ${ }^{55}$ )

BP, blood pressure; CVD, cardiovascular disease; eHEALS, e-health Literacy Scale; HDL, high-density lipoprotein; HLQ, Health Literacy Questionnaire; LDL, low-density lipoprotein; TC, total cholesterol.

\section{Statistical considerations}

For the primary outcome measure, assumptions used in the sample size and power estimates have been determined from TORPEDO data of 10181 routinely attending patients at moderate-to-high CVD risk. Calculations assume that $25 \%$ of people are meeting guidelinerecommended BP and LDL targets (as defined above) at baseline with mean systolic BP $136 \mathrm{~mm} \mathrm{Hg}$ (SD 17.2) and mean LDL cholesterol $2.5 \mathrm{mmol}$ (SD 0.70). A total sample size of 2000 participants, allowing for a $20 \%$ loss 
to follow-up, would have $90 \%$ power to detect an absolute improvement of at least $7.5 \%$ in the proportion of people meeting recommended targets using two-sided tests, with $\mathrm{p}$ values of less than 0.05 judged as significant. For secondary outcomes, this translates to a $2.8 \mathrm{~mm} \mathrm{Hg}$ absolute difference in systolic BP and a $0.11 \mathrm{mmol} / \mathrm{L}$ absolute difference in LDL cholesterol.

Although there is little literature on effect sizes of e-health interventions for CVD risk factors, we have powered the study on effect estimates that could be considered clinically meaningful for a moderate-to-high risk population. Based on previous work on modelling cardiovascular risk factor reductions, the BP and LDL cholesterol effect sizes mentioned above could each translate to around $5-10 \%$ RRR or combined RRR of $10-19 \%$ in cardiovascular events. ${ }^{56}$

Primary analyses will be unadjusted, following an intention-to-treat principle, and conducted blind to treatment allocation. If necessary, multivariate analyses will be performed to adjust for any significant differences between each study arm. Prespecified analyses will be conducted on the following subgroups: established CVD versus high-risk non-CVD; Aboriginal versus non-Aboriginal; proportion meeting/not meeting the primary endpoint at baseline; proportion adherent/not adherent to guideline-recommended BP and lipid medicines at baseline. Characteristics will be compared between groups using independent $\mathrm{t}$ tests for continuous variables and $\chi^{2}$ tests for categorical variables. Mean risk factor levels will be compared between groups in terms of relative risks, 95\% CIs and two-sided $\mathrm{p}$ values. Mann-Whitney $\mathrm{U}$ tests will be used where data are not normally distributed.

\section{Process evaluation}

Process evaluations explore the implementation, receipt and setting of an intervention and help in the interpretation of outcome results. ${ }^{57}$ Our team has extensive experience in such evaluations. ${ }^{44} 4858$ Analyses will be conducted by the Pawson and Tilley ${ }^{59}$ realistic evaluation model, which seeks to understand human actors' choices and actions, within the context of the systems in which these players operate. We will use mixed methods to investigate why the e-health strategy may or may not have been effective and which intervention components were most influential. Four data sources will be used: (1) quantitative data on patient measures described above; (2) usage of data extracted directly from the consumer portal; (3) patient and provider surveys of satisfaction, tool utility and health actions taken and (4) semistructured interviews with participants and care providers (including GPs) toward the end of study.

Multivariate subgroup analyses will assess for any differential impact of the intervention on outcome measures by Aboriginal status, postcode, income, education level, language spoken at home, age, gender and internet and mobile phone access. Qualitative data will explore participant views on benefits, disadvantages and acceptability of the portal. Taking an equity perspective, interviews will seek to understand barriers and enablers to uptake by particular subpopulations, including factors such as Internet/smartphone connectivity and geographical remoteness. To obtain a broad range of views, we will use a maximum variation sampling method based on patient demographics and health service characteristics. ${ }^{60}$ Sampling will continue until no new themes or categories emerge ('thematic saturation'). We anticipate, based on our previous experience, around 80 interviews will be required. In ACCHSs, Aboriginal participants will be interviewed by Aboriginal researchers. As with previous research collaborations, support and training will be provided for Aboriginal health researchers in the process evaluation, focusing not only on data collection but also on analysis and reporting. Analyses will be thematic and coding will be carried out inductively based on emergent themes. NVivo V.9 will be used to assist with interview data management. Given the focus of CONNECT on consumer engagement with an e-health strategy, assessment of health literacy (including communication with providers and understanding of information provided) will allow greater understanding and explanation of potential clinical outcomes as well as barriers and enablers that engage with the intervention. The process evaluation will provide important narratives on the role of e-health tools in the patient and care provider experience. These standalone research findings will make a novel contribution to translating findings into policy and practice.

\section{Economic evaluation}

A cost-effectiveness analysis will be undertaken to compare the e-health strategy with usual care. The economic evaluation will entail two components: a trialbased economic evaluation and a modelled economic evaluation of long-term costs and outcomes. The trialbased economic evaluation will estimate the incremental cost-effectiveness of the e-health strategy in terms of quality-adjusted life years (QALY) as measured over the follow-up period. This will enable an incremental cost per QALY gained to be estimated. The direct costs of the intervention over and above usual care, including training support and software maintenance, will be assessed. Alongside these will be cost offsets, in which the difference between the costs incurred in the utilisation of health services, medications and tests between treatment groups will be assessed. Data for these costs will be drawn from automated extracts of health service records (eg, service utilisation) and from self-reported questionnaires (eg, events, hospitalisations and quality of life). Details on medications, laboratory tests and service utilisation will be costed at prevailing rates. Hospitalisations will be costed using standard Australian National Diagnosis Related Groups (AN-DRG) cost weights.

A modelled economic evaluation will be built onto the trial to enable quality of life and survival to be examined 
over a longer time frame than the trial follow-up period. Patients in usual care and the e-health strategy would be tracked over this extended period to capture various health states (including death and various cardiovascular events). Transition across these health states will be based on probabilities in relation to long-term treatment effects, safety and disease progression derived from the trial findings and/or literature review. Data on costs and quality of life attached to various health states will also be drawn from literature review and trial data. With appropriate discounting, estimates of long-term costs and outcomes will fold out of the model. Sensitivity analyses will be conducted on variables such as discount rate, uncertainty in outcome estimates and assumptions made in costings. Different pricing scenarios will also be tested to determine threshold values for achieving costeffectiveness, including cost-effectiveness of different components of the strategy.

\section{ETHICS AND DISSEMINATION}

The findings of this study will be disseminated via the usual scientific forums including peer-reviewed publications and presentations at international conferences. The study will be administered by the George Institute for Global Health, with the design and conduct overseen by a Steering Committee. This committee has expertise in large-scale clinical trials and qualitative research, economic analysis, clinical CVD management and healthy policy implementation. This study will adhere to the National Health and Medical Research Council ethical guidelines for human research.

\section{CONCLUSION}

Less than half of all people at highest risk of a CVD event are receiving and adhering to best practice recommendations to lower their risk. In this project, we will examine the role of e-health-assisted consumer strategies as a means of overcoming such health system inefficiencies. With the availability of the PCEHR for all Australians in 2012, consumer-focused e-health is set to become a key component of the health system. Despite the scale of this initiative, uptake has been slow and there is little research on the factors that will support its uptake. Innovative strategies that are practical to implement and support negotiation of care between consumers and care providers are therefore urgently needed.

The CONNECT study will generate rigorously evaluated findings on an issue of national and international importance. The development of a content rich, consumer-focused e-health intervention that is fully integrated with the primary healthcare system will greatly inform the e-health agenda. Equitable access to these emerging technologies is essential, and this study, quantitatively and qualitatively, tests the acceptability and effectiveness of the intervention for Aboriginal and Torres Strait Islander peoples and other socioeconomically disadvantaged groups. The intervention will be compliant with Australian PCEHR specifications, thus allowing for seamless interoperability. This maximises its viability for large-scale implementation across Australia. If found to be successful, the CONNECT e-health strategy could be upscaled and expanded to increase compliance with international e-health strategies. The CONNECT strategy may also have applicability as a standalone strategy where electronic integration with primary care is unavailable.

\section{Author affiliations}

${ }^{1}$ The George Institute for Global Health, Sydney Medical School, University of Sydney, Sydney, Australia

${ }^{2}$ Sydney Medical School (Westmead), University of Sydney, Sydney, Australia

${ }^{3}$ University of New South Wales, Sydney, Australia

${ }^{4}$ Inala Indigenous Health Service, School of Medicine, University of

Queensland, Brisbane, Queensland, Australia

${ }^{5}$ University of Queensland, Brisbane, Queensland, Australia

${ }^{6}$ Centre for Health Informatics, Australian Institute of Health Innovation,

University of New South Wales, Sydney, New South Wales, Australia

${ }^{7}$ The George Institute for Global Health, Sydney Nursing School, University of Sydney, Sydney, Australia

${ }^{8}$ The George Institute for Global Health, Sydney, Australia

${ }^{9}$ Nuffied Department for Population Health, The George Institute for Global Health, Oxford University, Oxford, UK

${ }^{10}$ Faculty of Medicine, University of New South Wales, Sydney, Australia

Contributors JR and DP conceived the study and intervention, and drafted the protocol. TU, MFH, AR, NH, KP, CC, EH, AP and NZ contributed to the scientific design and protocol development. SJ led the economic analysis aspect of the design. AL, LN, GC and FH contributed to the practical components of the intervention design and delivery. All authors read and approved the final version of the manuscript.

Funding This work was supported by the National Health and Medical Research Council (Australia) for (APP1047508). JR is funded by a NHMRC/National Heart Foundation Postdoctoral Fellowship (632933). LN (APP1036763) and DP (APP1054754) are supported by a NHMRC Early Career Fellowships. AP (APP632938) and MH (APP1002600) are supported by an NHMRC Senior Research Fellowship.

\section{Competing interests None.}

Ethics approval University of Sydney Human Research Ethics Committee and the Aboriginal Health and Medical Research Council.

Provenance and peer review Not commissioned; peer reviewed for ethical and funding approval prior to submission.

Open Access This is an Open Access article distributed in accordance with the Creative Commons Attribution Non Commercial (CC BY-NC 3.0) license, which permits others to distribute, remix, adapt, build upon this work noncommercially, and license their derivative works on different terms, provided the original work is properly cited and the use is non-commercial. See: http:// creativecommons.org/licenses/by-nc/3.0/

\section{REFERENCES}

1. World Health Organisation: The top 10 causes of death. 2004 October 2008; Fact sheet N³10.http://www.who.int/mediacentre/ factsheets/fs310/en/index.html

2. Vos T, Barker B, Stanley L, et al. The burden of disease and injury in Aboriginal and Torres Strait Islander peoples 2003. Brisbane: University of Queensland, 2007.

3. Heeley E, Peiris D, Patel A, et al. Cardiovascular risk perception and the evidence practice gap in Australian General Practice (the AusHEART study). Med J Aust 2010;192:254-9.

4. Peiris D, Patel A, Cass A, et al. Cardiovascular disease risk management for Aboriginal and Torres Strait Islander peoples in primary health care settings-findings from the Kanyini Audit. Med J Aust 2009;191:304-9. 
5. Webster R, Heeley E, Peiris D, et al. Identifying the gaps in cardiovascular risk management. Med J Aust 2009;191:324-9.

6. Reid C, Nelson MR, Shiel L, et al. Australians at risk: management of cardiovascular risk factors in the REACH Registry. Heart Lung Circ 2008;17:114-18.

7. Wan Q, Harris MF, Jayasinghe UW, et al. Quality of diabetes care and coronary heart disease absolute risk in patients with type 2 diabetes mellitus in Australian general practice. Qual Saf Health Care 2006;15:131-5.

8. Steven I, Wing L. Control and cardiovascular risk factors of hypertension. An assessment of a sample of patients. Aust Fam Physician 1999;28:45-8.

9. Vale MJ, Jelinek MV, Best JD. How many patients with coronary heart disease are not achieving their risk-factor targets? Experience in Victoria 1996-1998 versus 1999-2000. Med J Aust 2002;176:211-15.

10. Chow CK, Jolly S, Rao-Melacini P, et al. Association of diet, exercise, and smoking modification with risk of early cardiovascular events after acute coronary syndromes. Circulation 2010;121:750-8.

11. Benner JS, Glynn RJ, Mogun H, et al. Long-term persistence in use of statin therapy in elderly patients. JAMA 2002;288:455-61.

12. Chan DS, Callahan CW, Hatch-Pigott VB, et al. Internet-based home monitoring and education of children with asthma is comparable to ideal office-based care: results of a 1-year asthma in-home monitoring trial. Pediatrics 2007:119:569-78.

13. Jan RL, Wang JY, Huang MC, et al. An internet-based interactive telemonitoring system for improving childhood asthma outcomes in Taiwan. Telemed J E Health 2007;13:257-68.

14. van der Meer V, van Stel HF, Detmar SB, et al. Internet-based self-management offers an opportunity to achieve better asthma control in adolescents. Chest 2007;132:112-19.

15. Grant RW, Wald JS, Schnipper JL, et al. Practice-linked online personal health records for type 2 diabetes mellitus: a randomized controlled trial. Arch Intern Med 2008;168:1776-82.

16. Stinson JN, Mcgrath PJ, Hodnett ED, et al. An internet-based self-management program with telephone support for adolescents with arthritis: a pilot randomized controlled trial. $J$ Rheumatol 2010;37:1944-52.

17. Green BB, Cook AJ, Ralston JD, et al. Effectiveness of home blood pressure monitoring, Web communication, and pharmacist care on hypertension control: a randomized controlled trial. JAMA 2008;299:2857-67.

18. Andrews G, Cuijpers P, Craske MG, et al. Computer therapy for the anxiety and depressive disorders is effective, acceptable and practical health care: a meta-analysis. PLOS ONE 2010;5:e13196.

19. Titov N, Andrews G, Schwencke G, et al. Randomized controlled trial of Internet cognitive behavioural treatment for social phobia with and without motivational enhancement strategies. Aust N Z J Psychiatry 2010;44:938-45.

20. Webb LT, Joseph J, Yardley L, et al. Using the internet to promote health behavior change: a systematic review and meta-analysis of the impact of theoretical basis, use of behavior change techniques, and mode of delivery on efficacy. $J$ Med Internet Res 2010;12:e4.

21. Wantland DJ, Portillo CJ, Holzemer WL, et al. The effectiveness of web-based vs. non-web-based interventions: a meta-analysis of behavioral change outcomes. J Med Internet Res 2004;6:e40.

22. van den Berg MH, Schoones JW, Vliet Vlieland TP. Internet-based physical activity interventions: a systematic review of the literature. $J$ Med Internet Res 2007;9:e26.

23. Balmford J, Borland R, Benda P. Patterns of use of an automated interactive personalized coaching program for smoking cessation. $J$ Med Internet Res 2008:10:e54.

24. Stevens JV, Funk LK, Brantley JP, et al. Design and implementation of an interactive website to support long-term maintenance of weight loss. J Med Internet Res 2008;10:e1.

25. Murray E, Burns J, See TS, et al. Interactive health communication applications for people with chronic disease. Cochrane Database Syst Rev 2005;(4):CD004274.

26. Free $C$, Knight R, Robertson S, et al. Smoking cessation support delivered via mobile phone text messaging (txt2stop): a single-blind, randomised trial. Lancet 2011;378:49-55.

27. Whittaker R, Borland R, Bullen C, et al. Mobile phone-based interventions for smoking cessation. Cochrane Database Syst Rev 2009; (4):CD006611.

28. Patrick K, Raab F, Adams MA, et al. A text message-based intervention for weight loss: randomized controlled trial. J Med Internet Res 2009;11:e1.

29. Hurling R, Catt M, Boni MD, et al. Using internet and mobile phone technology to deliver an automated physical activity program: randomized controlled trial. J Med Internet Res 2007;9:e7.
30. Petrie KJ, Perry K, Broadbent E, et al. A text message programme designed to modify patients' illness and treatment beliefs improves self-reported adherence to asthma preventer medication. Br J Health Psychol 2012;17:74-84.

31. Franklin VL, Waller A, Pagliari C, et al. A randomized controlled trial of Sweet Talk, a text-messaging system to support young people with diabetes. Diabet Med 2006;23:1332-8.

32. Marquez CE, de la Figuera von Wichmann M, Gil Guillen V, et al Effectiveness of an intervention to provide information to patients with hypertension as short text messages and reminders sent to their mobile phone (HTA-Alert). Aten Primaria 2004;34:399-405.

33. Miloh T, Annunziato R, Arnon R, et al. Improved adherence and outcomes for pediatric liver transplant recipients by using text messaging. Pediatrics 2009;124:e844-50.

34. Lester RT, Ritvo P, Mills EJ, et al. Effects of a mobile phone short message service on antiretroviral treatment adherence in Kenya (WelTel Kenya1): a randomised trial. Lancet 2010;376:1838-45.

35. Chow CK, Redfern J, Thiagalingam A, et al. Design and rationale of the tobacco, exercise and diet messages (TEXT ME) trial of a text message-based intervention for ongoing prevention of cardiovascular disease in people with coronary disease: a randomised controlled trial protocol. BMJ Open 2012;2:e000606.

36. Redfern J, Thiagalingam A, Jan S, et al. Development of a set of mobile phone text messages designed for prevention of recurrent cardiovascular events. Eur J Prev Cardiol 2012 [epub ahead of print]. doi: 10.1177/2047487312449416.

37. Tang PC, Ash JS, Bates DW, et al. Personal health records: definitions, benefits, and strategies for overcoming barriers to adoption. J Am Med Inform Assoc 2006;13:121-6.

38. Lau AY, Sintchenko V, Crimmins J, et al. Protocol for a randomised controlled trial examining the impact of a web-based personal health management system on the uptake of influenza vaccination rates. BMC Health Serv Res 2012;12:86

39. IOM (Institute of Medicine). Health IT and patient safety: building safer systems for better care. Washington, DC: The National Academies Press, 2012.

40. Australian Bureau of Statistics. Household use of information technology, Australia, 2010-11. Catalogue number 8146.0. Canberra: Commonwealth of Australia, 2011. http://www.abs.gov.au/ ausstats/abs@.nsf $/ \mathrm{mf} / 8146.0$

41. Australian Bureau of Statistics. National Aboriginal and Torres Strait Islander Social Survey, 2008. Cat. no.4714.0. Canberra: Commonwealth of Australia, 2010.

42. Australian Media Communications Authority. ACMA Communications report 2010-11. Canberra: Commonwealth of Australia, 2011.

43. Peiris $\mathrm{D}$, Usherwood $\mathrm{T}$, Panaretto $\mathrm{K}$, et al. The Treatment Of cardiovascular Risk in Primary care using Electronic Decision supOrt (TORPEDO) study - protocol for a cluster randomised, controlled trial of an electronic decision support and quality improvement intervention in Australian primary health care. BMJ Open 2012;2:e002177.

44. Peiris DP, Joshi R, Webster RJ, et al. An electronic clinical decision support tool to assist primary care providers in cardiovascular disease risk management: development and mixed methods evaluation. J Med Internet Res 2009;11:e51.

45. Peiris D, Usherwood T, Weeramanthri T, et al. New tools for an old trade: a socio-technical appraisal of how electronic decision support is used by primary care practitioners. Sociol Health IIIn 2011;33:1002-18.

46. National Vascular Disease Prevention Alliance. Guidelines for the assessment of absolute cardiovascular risk: National Vascular Disease Prevention Alliance, 2009.

47. PEN Computer Systems. Clinical Audit Tool. http://www.clinicalaudit. com.au/ (accessed 30 Aug 2013).

48. Neubeck L, Ascanio R, Bauman A, et al. Planning locally relevant internet programs for secondary prevention of cardiovascular disease. Eur J Cardiovasc Nurs 2011;10:213-20.

49. Fogg BJ. Persuasive technology: using computers to change what we think and do. USA: Morgan Kaufmann Publishers, 2003.

50. National Heart Foundation of Australia and the Cardiac Society of Australia and New Zealand. Reducing risk in heart disease: an expert guide to clinical practice for secondary prevention of coronary heart disease. Melbourne: National Heart Foundation of Australia, 2012. http://www.heartfoundation.org.au/SiteCollectionDocuments/ Reducing-risk-in-heart-disease.pdf

51. Osborne RH, Batterham RW, Elsworth GR, et al. The grounded psychometric development and initial validation of the Health Literacy Questionnaire (HLQ). BMC Public Health 2013;13:658.

52. Norman CD, Skinner HA. eHEALS: The eHealth Literacy Scale. $J$ Med Internet Res 2006;8:e27. 
53. World Health Organisation. Global Physical Activity Questionnaire (GPAQ) Analysis Guide. 2011. http://www.who.int/chp/steps/GPAQ/ en/index.html (accessed 14 Jun 2013).

54. West R, Hajek P, Stead L, et al. Outcome criteria in smoking cessation trials: proposal for a common standard. Addiction 2005;100:299-303.

55. Viney R, Norman R, King MT, et al. Time trade-off derived EQ-5D weights for Australia. Value Health 2011;14:928-36.

56. Pill Collaborative Group. An international randomised placebocontrolled trial of a four-component combination pill ("Polypill") in people with raised cardiovascular risk. PLoS ONE 2011;6:e19857.
57. Oakley A, Strange V, Bonell C, et al. Process evaluation in randomised controlled trials of complex interventions. $B M J$ 2006;332:413-16.

58. Jan S, Usherwood T, Brien JA, et al. What determines adherence to treatment in cardiovascular disease prevention? Protocol for a mixed methods preference study. BMJ Open 2011;1: e000372.

59. Pawson R, Tilley N. Realistic evaluation. London; Thousand Oaks: Sage, 1997.

60. Patton M. Qualitative research and evaluation methods. USA: SAGE Publications, 2002. 